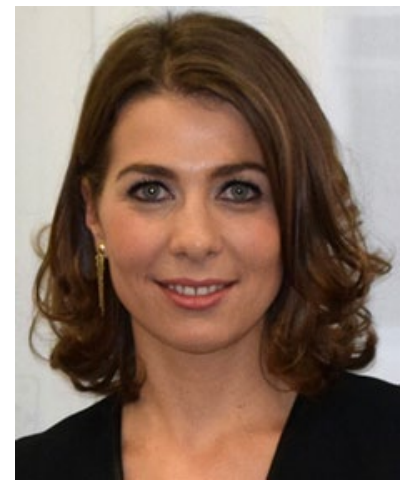

Eva-Luise Hobl

\section{Eva-Luise Hobl ist Researcher of the Month, Dezember 2014}

Die Jury „Researcher of the Month“ verleiht die Auszeichnung für den Monat Dezember 2014 an Eva-Luise Hobl von der Universitätsklinik für Klinische Pharmakologie. Anlass für die Auszeichnung ist die im Top-Journal "Journal of the American College of Cardiology (JACC)“ (IF 15.343) erschienene Arbeit: „Morphine Decreases Clopidogrel Concentrations and Effects: A Randomized, Double Blind, Placebo-Controlled Trial“"

Die Arbeit entstand unter der Leitung von Bernd Jilma an der Universitätsklinik für Klinische Pharmakologie in Kooperation mit der Klinischen Abteilung für Medizinisch-Chemische Labordiagnostik (Thomas Stimpfl) sowie dem Institut für Medizinische Statistik (Martin Posch).

\section{Morphin vermindert die Konzentration und Wir- kung von Clopidogrel}

Die Verabreichung des P2Y12-Inhibitors Clopidogrel mit Aspirin ist eine wichtige Kombination zur Behandlung des Akuten Koronarsyndroms.

Obwohl in den Richtlinien die Gabe von Morphin zur Schmerzbehandlung empfohlen wird, gibt es keine Daten aus randomisiert-kontrollierten klinischen Studien, die einen Benefit von Morphin belegen. Die Gabe von Morphin ist bei PatientInnen mit Nicht-ST-Hebungsinfarkt sogar mit einer höheren Mortalität verbunden. Ein möglicher kausaler Zusammenhang könnte dadurch erklärt werden, dass Opiate die Magenentleerung verlangsamen und somit die Absorption von oral verabreichten Medikamenten verzögern, was in weiterer Folge zu verminderten Wirkstoffspiegeln führen kann.

Das Ziel dieser Studie war es zu prüfen, ob Morphin die Plasmakonzentration des aktiven Metaboliten und die plättchenfunktionshemmende Wirkung von Clopidogrel im Rahmen einer Arzneimittelwechselwirkung reduziert. Die Ergebnisse zeigen, dass Morphin die Aufnahme von Clopidogrel um 30 min. verzögert und die Plasmakonzentrationen des aktiven Clopidogrelmetaboliten um bis zu $50 \%$ vermindert. Die Verabreichung von Morphin erzeugte ein pharmakokinetisches Profil, welches mit dem „Poor Metabolizer“-Phänotypus vergleichbar ist. Im Vergleich zu Placebo wurde unter Morphin die Zeit bis zum Eintritt der maximalen Thrombozytenaggregationshemmung verdoppelt (1,5 vs. $3 \mathrm{~h}$ ), in einigen Probanden wurde die maximale Wirkung sogar erst nach $5 \mathrm{~h}$ erreicht.

\section{Welche Auswirkungen haben diese Ergebnisse auf die klinische Praxis?}

Eine schnelle und effiziente Hemmung der Plättchenfunktion ist ein zentraler Faktor in der Therapie des Akuten Koronarsyndroms. Durch Morphingabe wurde das Ansprechen auf Clopidogrel um circa zwei Stunden verzögert, was den Behandlungserfolg ebenfalls negativ beeinflussen kann. Zusammenfassend verzögert Morphin die Aufnahme von Clopidogrel, vermindert die Plasmakonzentration des aktiven Metaboliten und reduziert die Wirkung von Clopidogrel, wodurch es unter Umständen zu einem Therapieversagen im Rahmen des Akuten Koronarsyndroms kommen kann.

Eva-Luise Hobl wurde für die vorliegende Arbeit mehrfach international ausgezeichnet: Im November 2013 wurde ihr von der American Heart Association der „Samuel A. Levine Young Clinical Investigator Award“ verliehen. Im Februar 2014 wurde die Arbeit als „Best Clinical Abstract“ beim Annual Meeting der Gesellschaft für Thrombose- und Hämostaseforschung ausgezeichnet. Im Juni 2014 erhielt sie den „EPHAR Young Clinical Investigator Award" der Federation of European Pharmacological Societies. 